\title{
Operation of the sympathetic magical law of contagion in interpersonal attitudes among Americans
}

\author{
PAUL ROZIN, CAROL NEMEROFF, MARCIA WANE, and AMY SHERROD \\ University of Pennsylvania, Philadelphia, Pennsylvania
}

\begin{abstract}
The law of contagion is one of the laws of sympathetic magic put forth about 100 years ago to account for magical belief systems in traditional cultures. This law holds that when two objects (usually animate) come into contact with each other, there is a potentially permanent exchange of properties between them. In a prior study, we demonstrated that this law fits well with a variety of behaviors of Americans in response to disgusting and dangerous objects, primarily in the food domain. In the present study, we explored by questionnaire the operation of this principle in the interpersonal domain. We examined two dimensions of contagion. First, we show that the history of human contacts with an object can affect the value of that object both positively and negatively, depending on the nature of one's relation to its contact source. Second, we report results consistent with a belief in both "forward" action (in which influence passes from source to recipient) and "backward" action (whereby acting on a person's possessions or bodily residues can influence that individual). We conclude that contagion is an operative belief in American culture.
\end{abstract}

The law of contagion is one of three laws of sympathetic magic discussed in the anthropological literature. These laws were initially propounded by E. B. Tylor (1871/ 1974), were expanded upon by Sir James Frazer (1890/ 1959), and were conceptually elaborated by Marcel Mauss $(1902 / 1972)$. These laws were presented as general beliefs (not necessarily explicit) that underlie diverse magical practices and rituals in traditional cultures. These authors thought of the laws of sympathetic magic (contagion, similarity, and opposition) as laws of thought, and recognized their similarity to the laws of association (contiguity, similarity, and opposites). However, they noted a major difference: whereas the laws of association are merely laws of sequencing of thought, the laws of sympathetic magic are assumptions about the physical world as well.

According to the law of contagion, things that once have been in contact with each other may continue to influence each other, through the transfer of some or all of their properties. This influence remains after the physical contact has ceased, and may be permanent ("once in contact, always in contact"). We may conceive of this transfer of properties as being accomplished by the passing of

This research was supported by funds from the John D. and Catherine T. MacArthur Foundation Mental Health Research Network on Determinants and Consequences of Health-Promoting and Health-Damaging Behaviors. Our thanks to Clark McCauley for critical comments on the manuscript. Carol Nemeroff's current address is Department of Psychology, Arizona State University, Tempe, Arizona 85281. Correspondence may be addressed to Paul Rozin, Department of Psychology, University of Pennsylvania, 3815 Walnut St., Philadelphia, PA 19104-6196. some contagious entity, or "essence," between source and recipient (see Rozin \& Nemeroff, in press, for a review).

In our analysis, the laws of sympathetic magic have two basic dimensions. First, the "magic" can be positive or negative. Thus, in contagion, contact of an object with a loved, respected, or revered person can enhance the value of the object or render it beneficial (positive contagion), whereas contact with a disliked, despised, or feared person can devalue the object or render it dangerous (negative contagion). Second, transmitted essence can mediate effects either in its source or in its recipient. In what we call "forward contagion," the essence influences the entity it ultimately contacts (the recipient). In "backward contagion," action on the essence contained in a bodily residue acts back on its source, as in the practice of scorching a lock of an enemy's hair to cause harm. Although there is a rational analogue for forward contagion (i.e., microbial contamination), backward contagion has no analogue in terms of known physical principles.

In a previous paper (Rozin, Millman, \& Nemeroff, 1986), we explored two of the laws of magic, contagion and similarity, and presented evidence that they are operative in Americans in cases of food rejection and disgust. The interpersonal domain is the area in which the law of contagion is most salient in traditional cultures. The present study explores the operation of contagion in the thinking of contemporary Americans in the domain of interpersonal relations, and explicitly probes both the positive/negative and the forward/backward dimensions.

\section{METHOD}

The subjects were 140 adult volunteers from the University of Pennsylvania Community $(N=104)$, students at Shippensburg University 
in south-central Pennsylvania $(N=26)$, and members of a hospital office staff in Philadelphia $(N=10)$. There were 74 males and 66 females, with a mean age of 22.6 years (range 16-57). The questionnaire was anonymous and was filled out either in class or privately. The subjects indicated their age, sex, race, religion, and whether they were currently in a romantic relationship. The remaining questions explored contagion beliefs.

Six objects were described as coming into contact with four different persons (sources), designated FRIEND, LOVER, DISLIKE, and UNSAVORY. The subjects identified these four persons initially according to the following instructions: "a best friend (the person you feel closest to, nonromantically); boyfriend/ girlfriend/ lover/ spouse (the person you feel closest to, romantically); a disliked peer (the person you dislike most from your peer group); and an unsavory person (an unsavory character, a bum, or streetperson)." The subjects who were not currently in a romantic relation were asked not to answer the LOVER question for each object.

The subjects' answers were in the form of ratings on a 200-point scale running from -100 to +100 . Zero was neutral, +100 was "the most pleasant thing you can imagine," and -100 was "the most unpleasant thing you can imagine." The question was, "How would you feel about (eating, wearing, etc....)."

For the first four objects, the subjects also rated a control entity (an identical object that had not come into contact with any source) to obtain a baseline for the judgment-of-contagion effects. For the last two objects, there was no obviously meaningful control, so the baseline was assumed to be zero. The objects and questions were as follows (with the abbreviated designations in capital letters).

1. SWEATER. "How would you feel about wearing a particular brand-new sweater of a style you find very attractive, that is thoroughly laundered but has never been worn?"' (NEW, CONTROL). "Consider another instance of this same sweater, that is thoroughly laundered and that once belonged to each of the persons listed below. In each case, rate how you would feel about wearing the sweater." (FRIEND, LOVER, DISLIKE, UNSAVORY).

2. HAMBURGER. "How would you feel about taking a bite out of a hamburger at exactly the same place where a small, bite-shaped piece had been cut out with a clean knife?" (UNBIT, CONTROL). "How would you feel about taking a bite out of a hamburger at exactly the place where each of the following persons had already taken a bite? (Think of a brand-new hamburger for each person. The person just took the bite, but you didn't see them and they are not present.)"

3. APPLE. As above, replacing hamburger with apple.

4. HAIRBRUSH (GET, FORWARD). "How would you feel about brushing your hair with a brand-new hairbrush?”' (NEW, CONTROL). "How would you feel about brushing your hair with a thoroughly cleaned hairbrush that once belonged to and was used by each of the following persons?"

5. HAIRBRUSH (GIVE, BACKWARD). "How would you feel about your thoroughly cleaned hairbrush being given to each of the following persons? Each would also be told your name. Assume you will never use the brush again." (No control for this item.)

6. HAIR (GIVE, BACKWARD). "Imagine a lock of your hair that was cut off at your last visit to a barber/hairdresser. How would you feel about it being given to each of the following persons. Each person will be told your name." (No control for this item.)

This set of questions was designed to explore the dimensions of contagion as follows: Of the 24 items (not including controls), 12 were meant to be negative (DISLIKE, UNSAVORY $\times$ all objects) and 12 positive (FRIEND, LOVER $\times$ all objects); 16 dealt with forward causation (first 4 objects $\times$ all sources) and 8 with backward causation (last 2 objects $X$ all sources) (Table 1$)$.

\section{RESULTS}

For questions with controls, the basic measure of a contagion effect was the difference in ratings between a con- trol object and the corresponding object that had contacted a source. A negative value indicated a negative contagion effect (reduction in value), and a positive value indicated a positive contagion effect (enhancement in value). For the items without controls (HAIRBRUSH-BACKWARD and HAIR), raw ratings were used.

The percent of subjects who showed a substantial contagion effect of at least a 20-point departure from baseline is shown in Table 1. In addition, one-way ANOVAs were carried out for each object using source as the group factor. Only the 91 subjects who were currently in a romantic relationship were included in the sample for the ANOVAs. The main effect of contact with sources was highly significant (well below .001) for all objects [minimum $F(3,88)=43.92]$. However, further individual $t$-tests for each person-object pairing showed that the effect of person varied with the object. Whereas 17 of the 24 items probed showed significant contagion effects at the .001 level, the effect of two of these objects was in a direction opposite of that predicted. Both objects involved small negative responses to contagion from FRIEND (SWEATER and HAIRBRUSH-FORWARD). Similarly, six of the seven items that failed to show significant effects at all involved FRIEND and LOVER; average scores on these items represented not only positive and neutral ratings, but also a substantial number of negative ratings (see the two columns on the right in Table 1).

In general, in cases of forward contagion (in which the subject used items contacted by others), the negative effects of contact with negative sources were far more consistent and far outweighed the enhancing effect of contact with positive sources. Thus, the mean effect of contact with DISLIKE and UNSAVORY across the

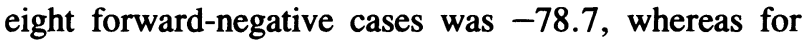
FRIEND and LOVER the variability in the valence of responses resulted in a mean effect of -3.0 . In contrast, in backward-contagion cases, the subjects seemed more positive about their residues falling into the hands of friends or lovers than they were negative about them falling into the hands of disliked or unsavory people.

The relative magnitude of negative reactions to the two negative sources, DISLIKE and UNSAVORY, depended on the object. For backward-contagion cases, since the disliked person was likely to have negative motivations toward the subject, an account in terms of magical contagion would predict more concern about the disliked person's possession of a personal object, compared with possession of that object by an unknown, unsavory person. In both of the backward-contagion cases, the DISLIKE scores were indeed more negative than the UNSAVORY scores. On the other hand, for forward-contagion cases (SWEATER, HAMBURGER, APPLE, and BRUSHFORWARD), in which disease transfer was a likely concern for the subject, the negative effect was greater for unsavory persons. No such object dependence occurred 
Table 1

Incidence of Magical Beliefs

\begin{tabular}{|c|c|c|c|c|c|c|}
\hline Question & Valence & Direction & $M$ & $S D$ & $\leq-20 \dagger$ & $\geq 20 \ddagger$ \\
\hline \multicolumn{7}{|l|}{ SWEATER } \\
\hline FRIEND-NEW & + & For & $-7.4^{*}$ & 24.3 & 22.1 & 8.6 \\
\hline LOVER-NEW & + & For & 1.6 & 17.0 & 10.5 & 13.7 \\
\hline DISLIKE-NEW & - & For & $-74.2^{*}$ & 54.2 & 82.1 & 0 \\
\hline UNSAVORY-NEW & - & For & $-104.1^{*}$ & 60.1 & 95.0 & 0.7 \\
\hline \multicolumn{7}{|l|}{ HAMBURGER } \\
\hline FRIEND-PLAIN & + & For & -4.2 & 39.7 & 20.1 & 15.1 \\
\hline LOVER-PLAIN & + & For & 7.6 & 30.1 & 8.4 & 27.4 \\
\hline DISLIKE-PLAIN & - & For & $-55.4^{*}$ & 47.9 & 77.7 & 1.4 \\
\hline UNSAVORY-PLAIN & - & For & $-89.6 *$ & 50.2 & 93.5 & 1.4 \\
\hline \multicolumn{7}{|l|}{ APPLE } \\
\hline FRIEND-PLAIN & + & For & -7.2 & 35.9 & 20.1 & 7.2 \\
\hline LOVER-PLAIN & + & For & -0.2 & 29.7 & 9.2 & 14.3 \\
\hline DISLIKE-PLAIN & - & For & $-59.9 *$ & 51.1 & 79.9 & 2.1 \\
\hline UNSAVORY-PLAIN & - & For & $-94.8^{*}$ & 53.0 & 94.2 & 0.7 \\
\hline \multicolumn{7}{|c|}{ HAIRBRUSH (GET, FOR) } \\
\hline FRIEND-NEW & + & For & $-9.9 *$ & 28.2 & 20.7 & 3.6 \\
\hline LOVER-NEW & + & For & -3.9 & 22.0 & 11.3 & 6.2 \\
\hline DISLIKE-NEW & - & For & $-56.9 *$ & 55.7 & 70.0 & 0.7 \\
\hline UNSAVORY-NEW & - & For & $-94.6 *$ & 62.6 & 88.6 & 0 \\
\hline \multicolumn{7}{|c|}{ HAIRBRUSH (GIVE, BACK) } \\
\hline FRIEND & + & Back & $23.8 *$ & 36.9 & 2.1 & 37.1 \\
\hline LOVER & + & Back & $26.0 *$ & 37.7 & 2.1 & 38.1 \\
\hline DISLIKE & - & Back & $-12.6^{*}$ & 35.2 & 32.1 & 7.8 \\
\hline UNSAVORY & - & Back & -8.5 & 42.8 & 26.4 & 13.6 \\
\hline \multicolumn{7}{|l|}{ HAIR (GIVE, BACK) } \\
\hline FRIEND & + & Back & $12.4^{*}$ & 33.4 & 4.3 & 28.0 \\
\hline LOVER & + & Back & $26.0^{*}$ & 38.0 & 2.2 & 45.6 \\
\hline DISLIKE & - & Back & $-22.9^{*}$ & 35.1 & 38.1 & 2.9 \\
\hline UNSAVORY & - & Back & $-20.6^{*}$ & 34.9 & 33.1 & 2.9 \\
\hline
\end{tabular}

Note $-M=$ mean. $S D=$ standard deviation. Valence $=$ positive or negative source. Direction $=$ forward (For) or backward (Back) influence. $N=138-140$ for all items except LOVER; $N=90-91$ for LOVER. $\quad{ }^{*} p<.001$. †Percent of subjects scoring $\leq-20 . \quad \ddagger$ Percent of subjects scoring $\geq 20$.

for LOVERS versus FRIENDS; across all items, the mean magical effect was an enhancement of 9.5 points for LOVERS and 1.2 points for FRIENDS, with the small difference favoring LOVERS consistent across items.

\section{DISCUSSION}

In this largely college-based sample, both positive and negative contagion effects occurred for every object explored. There was a general tendency for negative contagion to be more salient than positive contagion for cases of forward action, to the point that, even with ostensively positive sources (lovers and best friends), more subjects showed negative than positive effects. In our sample, the predominance of negative effects for positive sources in forward-contagion scenarios may be the result of a culturally determined competition of disgust and germ concern with positive feelings about certain objects that have come into contact with a lover or friend. Consistent with this hypothesis, in backward-contagion cases, in which germ theory does not apply, the negative bias and the variability of results disappear. However, the dominance of negative interpersonal contagion is suggested as well by the anthropological literature on cultures that lack germ theory; interpersonal pollution is widely reported in these societies, whereas transvaluation (enhancement) is a relatively rarely documented phenomenon.

Backward contagion is of particular concern in understanding magical beliefs and in differentiating them from simple associations (Rozin et al., 1986; Rozin \& Nemeroff, in press). Although associative principles might predict that one would feel bad about an enemy's having a lock of one's hair, they do not predict the belief or feeling that one could come to harm in this way. Our findings do not actually indicate a belief in backward causation; they simply report effects consistent with it under suitable conditions. Although we cannot, at this point, eliminate the associative interpretation, contagion accounts well for certain features of people's reactions to situations that involve contact and are difficult to explain by the associative view. For instance, why would many individuals become more upset by wearing an innocent-looking sweater that was once worn by Adolph Hitler than by holding a book written about him, with his name and picture all over the cover and the story of his life inside? The explanation of "stronger association" begs the question of why brief contact should result in such strong associations. Moreover, contiguity focuses on temporal or spatial proximity rather than the physical contact that is critical in magical contagion. In considering our results, it seems unlikely that these effects would have obtained if the questions had described mere proximity of source to object, rather than actual contact.

We take the results of our questionnaire at face value as representing the attitudes of our subjects. Our concern with demand characteristics is mitigated by our observations of abundant examples of contagion in everyday thinking and behavior in this culture, including attachments to objects related to one's family history, the high value placed on former possessions of famous people, and hysteria about contact with AIDS victims. We view the magical law of contagion as a significant influence in people's reactions to objects in the world. 


\section{REFERENCES}

Frazer, J. G. (1959). The new golden bough: $A$ study in magic and religion (Abridged; T. H. Gaster, Ed.). New York: Macmillan. (Original abridged work published 1922; original work published 1890)

Mauss, M. (1972). A general theory of magic. (R. Brain, Trans.). New York: Norton. (Original work published 1902)

Rozin, P., Millman, L., \& Nemeroff, C. (1986). Evidence for the operation of the laws of sympathetic magic in disgust and other domains. Journal of Personality \& Social Psychology, 51, 703-712.
Rozin, P., \& Nemeroff, C. (in press). The laws of sympathetic magic: A psychological analysis of similarity and contagion. In J. Stigler, G. Herdt, \& R. A. Shweder (Eds.), Culture and human development. Cambridge, England: Cambridge University Press.

TyLOR, E. B. (1974). Primitive culture: Researches into the development of mythology, philosophy, religion, art and custom. New York: Gordon Press. (Original work published 1871)

(Manuscript received December 24, 1988.) 